\title{
Robotics assistance in neurosurgery-improving the outcome for our patients
}

\author{
Jesus Lafuente ${ }^{1}$ (D) \\ Received: 13 July 2018 / Accepted: 17 July 2018 /Published online: 14 August 2018 \\ (C) Springer-Verlag GmbH Austria, part of Springer Nature 2018
}

As scientists, neurosurgeons try to keep up with all innovations that are presented to us with the view of making our life easier and more importantly improve the outcome of our patients.

Robotic technology, inherited from the military huge resources for new technologies, as occurred with ultrasonography in the past, provides another great tool to improve our surgical techniques. As medicine inherited some of these military techniques, medical investment companies had invariably improved this technology for medical applications, and robotics is no exception. However, current robotics systems are assistive and lack of real autonomy.

Robotics has a wide range of surgical applications allowing finer movements when dealing with extraction of lesions such as in a prostatectomy, but also showing great accuracy when placing electrodes in deep-seated brain structures, bypassing vessels and functional brain areas for epilepsy mapping, and therefore minimizing surgical complications. Also in neurosurgery, Robotics has also been used to perform burr holes and craniotomies.

But I suspect that the questions here are:

Can we use robotics for all neurosurgical procedures?

How far can we get with this technology?

Robotics, to place electrodes in the brain, is feasible, and accuracy is better than with current navigation systems; however, it has the same limitation as the navigation systems with respect to the "brain shift" effect, if one makes a craniotomy to place deep electrodes, intending to leave also a cortical grid.

This article is part of the Topical Collection on Spine - Other

Jesus Lafuente

jlbspine@gmail.com

1 Department of Neurosurgery, Parc de Salut Mar, Barcelona, Spain
However, if only placing deep electrodes, these are done individually and through a very small hole, rendering the brain shift effect nonexistent.

However, if the intention is to use robotics to remove lesions within the brain or the spinal cord, I think at present the technology is not able to do this. The current robotic systems are too bulky and have limited range of movements to get into the brain and remove lesions or clip aneurisms.

In the other hand, it looks that there is a role of robotics in spine surgery, as bone is a fixed structure and is easy to place pedicle screws assisted with navigation systems, from cervical spine to sacrum. Lumbar anterior approach has also been tried through the peritoneum, only in cadaver, taking advantage of the expandable peritoneal cavity, to perform discectomies and corpectomies. This has shown some limitations at the level of L5/S1 due to the promontory angle. Similarly, robotic technology has shown good potential in transoral surgery, particularly closing the posterior pharyngeal wall, which is a crucial step to avoid CSF fistula and its potential complications.

At the present time, robotics in neurosurgery is used as an assisting and accuracy tool used mainly for placement of deepseated brain electrodes or perform a burr hole in the skull, and to place pedicle screws in the spine from cervical to lumbosacral.

As medical robotic systems become established in clinics around the world, one of the next paradigm shifts in the field will come from more precise, less invasive, targeted systems, known as micro- and nanorobotics. Micro- and nanorobots are small, motile devices that convert chemical or/and physical energy into mechanical actuation and have the potential to enable minimally invasive treatment and targeted drug/cell delivery with high precision.

Looks like we are still at early stages in robotics for neurosurgical procedures of its full potential, and it is clear to me that thanks to the determination of many of our colleagues vision, time, and technical preparation, this technology will get to its full potential and I believe we will, in the near future, be able to enter the brain with robotic technology. 